\title{
Perceptions, Practices, and Mother's Willingness to Provide Meconium for Use in the Assessment of Environmental Exposures among Children in Mukono and Pallisa Districts, Uganda
}

\author{
John C. Ssempebwa ${ }^{D},{ }^{1}$ Geofrey Musinguzi $\mathbb{D}^{1},{ }^{1}$ and Simon Peter Sebina Kibira ${ }^{2}$ \\ ${ }^{1}$ Department of Disease Control and Environmental Health, School of Public Health, College of Health Sciences, \\ Makerere University, Kampala, Uganda \\ ${ }^{2}$ Department of Community Health and Behavioural Sciences, School of Public Health, College of Health Sciences, \\ Makerere University, Kampala, Uganda
}

Correspondence should be addressed to John C. Ssempebwa; jssemps@musph.ac.ug

Received 13 February 2018; Accepted 7 June 2018; Published 18 July 2018

Academic Editor: Julio Diaz

Copyright (C) 2018 John C. Ssempebwa et al. This is an open access article distributed under the Creative Commons Attribution License, which permits unrestricted use, distribution, and reproduction in any medium, provided the original work is properly cited.

\begin{abstract}
Presence of biomarkers or metabolites is assessed in various human biospecimens including meconium in the investigation of exposures to environmental contaminants. This study gathered data on the perceptions and practices of mothers in two rural districts of Uganda concerning meconium and their willingness to provide meconium from their babies for research purposes. The study reveals a wide range of perceptions and beliefs around meconium as well as a number of associated taboos and practices. Many participants noted that meconium could be used to detect ailments among newborns based on its appearance. Practices and beliefs included using it to prevent stomach discomfort and other ailments of newborns, as a means to confirm paternity and initiate the child into the clan as well as facilitating father-child bonding that included ingestion of meconium by the fathers. Most mothers indicated scepticism in accepting to provide meconium for research purposes and had fears of unscrupulous people disguising as researchers and using meconium to harm their children. However, some were willing to provide meconium, if it helped to detect ailments among their children. These perceptions and practices may negatively influence mothers' willingness to participate in meconium study. However, through provision of educational and behaviour change interventions, mothers' willingness to participate in a meconium study can be improved.
\end{abstract}

\section{Introduction}

Presence of biomarkers and/or metabolites is assessed in various human tissues in the investigation of exposures to environmental contaminants. Among the human tissues are blood $[1,2]$, urine $[3,4]$, breast milk $[5,6]$, and meconium [7-9]. Meconium testing provides a more sensitive matrix to measure fetal exposure to toxicants, due to its ability to cover a longer period of exposure than other matrices such as infant hair and cord blood [10]. Meconium starts to accumulate in the $13-16^{\text {th }}$ week of pregnancy and it is passed by a newborn within the first $24-48$ hours soon after birth
$[11,12]$. Meconium is composed of materials ingested during the time the infant spends in the uterus. It is a cumulative biological matrix of prenatal toxicant exposure, and its analysis has been documented as a sensitive, diagnostic tool to detect fetal exposure to heavy metals, pesticides, and other contaminants $[10,13,14]$. Meconium collection is easy and noninvasive; therefore it holds promise as a biological matrix for measuring the intensity and duration of environmental toxicant exposure.

Meconium provides key essential information about fetal exposure to toxic substances and may provide the groundwork for protecting the newborn from further damage $[15,16]$. 
In many African countries, there are taboos associated with fecal matter and therefore the use of meconium as a biomarker would be faced with some cultural challenges. Communities, traditions, and cultures have different practices, values, and beliefs surrounding handling of meconium [17-19] and this might affect mothers' willingness to participate in meconium testing interventions or allow the use of meconium for research purposes. Culture plays a major role in the way a woman perceives and prepares for her birthing experience. Each culture has its own values, beliefs, and practices related to pregnancy and birth [20-22]. Meconium testing is feared to have social risks particularly for mothers, especially when it involves testing for alcohol and any other drug use [18, 23, 24]. Fear, embarrassment, and guilt have been reported to contribute to low consent rates of mothers to participate in meconium screening programs $[8,23]$.

Understanding whether mothers would be willing to allow the use of their newborn infant meconium for research purposes has the potential to inform future meconium studies especially in low income countries where there are many cultural beliefs and practices surrounding births $[19,25]$. In this study, we explored mothers' perceptions, beliefs, practices, and willingness to participate in research where collection and use of meconium from newborns would be conducted in studying environmental exposures. The study was conducted in two districts of Uganda, Mukono in the central and Pallisa in the eastern part of the country.

The objectives of the study were to explore the sociocultural aspects including practices, perceptions, and beliefs surrounding meconium and to determine the willingness of mothers to participate in research where meconium would be used to assess exposure to environmental contaminants. The study findings may help identify reasons that could encourage or prevent mothers' participation in research where meconium is used to assess environmental exposures.

\section{Methods}

2.1. Study Design and Setting. This was an ethnographic study conducted in selected health centres (HC) and in the general community of Mukono and Pallisa districts among women of childbearing age. Mukono is located in central Uganda with 13 subcounties while Pallisa is located in eastern Uganda and has 14 subcounties (Figure 1). The study participants were recruited from four randomly selected subcounties in each of the two districts: Goma, Kyampisi, Mukono Town Council, and Nama in Mukono district and Butebo, Kibale, Pallisa Town Council, and Kakoro in Pallisa district.

The basic characteristics of the mothers who participated in the in-depth interviews are summarized in Table 1; majority of the mothers were aged 18-30 years, had no education or had only attained primary, were Catholics, and were married.

2.2. Data Collection, Management, and Analysis. Participants were purposely selected to provide details about their experiences. The team conducted 30 key informant (KI) interviews with health workers at selected health centres, Village Health Team (VHT) members, trained traditional birth attendants (TBAs), and community leaders. Forty-five (45) in-depth
TABLE 1: Sociodemographic characteristics of the mothers in the indepth interviews.

\begin{tabular}{lc}
\hline Variables & Total Number (\%) \\
\hline Age Category & $14(31.1)$ \\
$18-24$ & $14(31.1)$ \\
$25-30$ & $13(28.9)$ \\
$31-35$ & $4(8.9)$ \\
$36-40$ & \\
Education level attained & $33(73.4)$ \\
Primary/No education & $7(15.6)$ \\
Lower secondary (S.1-S.4) & $2(4.4)$ \\
Higher secondary (S.5-S.6) & $3(6.7)$ \\
Tertiary & \\
Religion & $14(31.1)$ \\
Catholic & $13(28.9)$ \\
Anglican & $12(26.7)$ \\
Moslem & $5(11.1)$ \\
Pentecostal/Born again & $1(2.2)$ \\
Others & \\
Marital Status & $43(95.6)$ \\
Married & $2(4.4)$ \\
Not married &
\end{tabular}

interviews (IDIs) were also held with pregnant women and mothers who had birthing experience. The interviews lasted approximately 60 minutes. All the interviews were conducted in the local languages (Luganda in Mukono district and Lugwere in Pallisa district) and audio recorded. The KI and IDI guides were pretested in nonstudy villages in both Mukono and Pallisa to improve understanding and relevance and to elicit better responses. These were modified accordingly based on the feedback.

All audio files were transcribed verbatim and translated to English. The initial codes were derived from reading the transcripts and these were used to develop a codebook that was applied to all the transcripts. The new codes that emerged during the coding process were also added to the codebook. All codes were refined after consensus among the team. The codes were grouped into categories to give meaning to the data. These were then categorised under the relevant themes that reflected the objectives of the study.

2.3. Ethical Considerations. Approval to conduct the research was obtained from the Makerere University School of Public Health, Higher Degrees, Research and Ethics Committee and from the Uganda National Council for Science and Technology. Written informed consent was obtained from each participant, following a detailed explanation of the research purpose.

\section{Results}

3.1. Knowledge and Perceptions on Meconium. Mothers were asked to describe the baby's first stool (meconium) in terms of colour, smell, and texture. They described meconium as being blackish, charcoal black, greenish, or yellowish in 


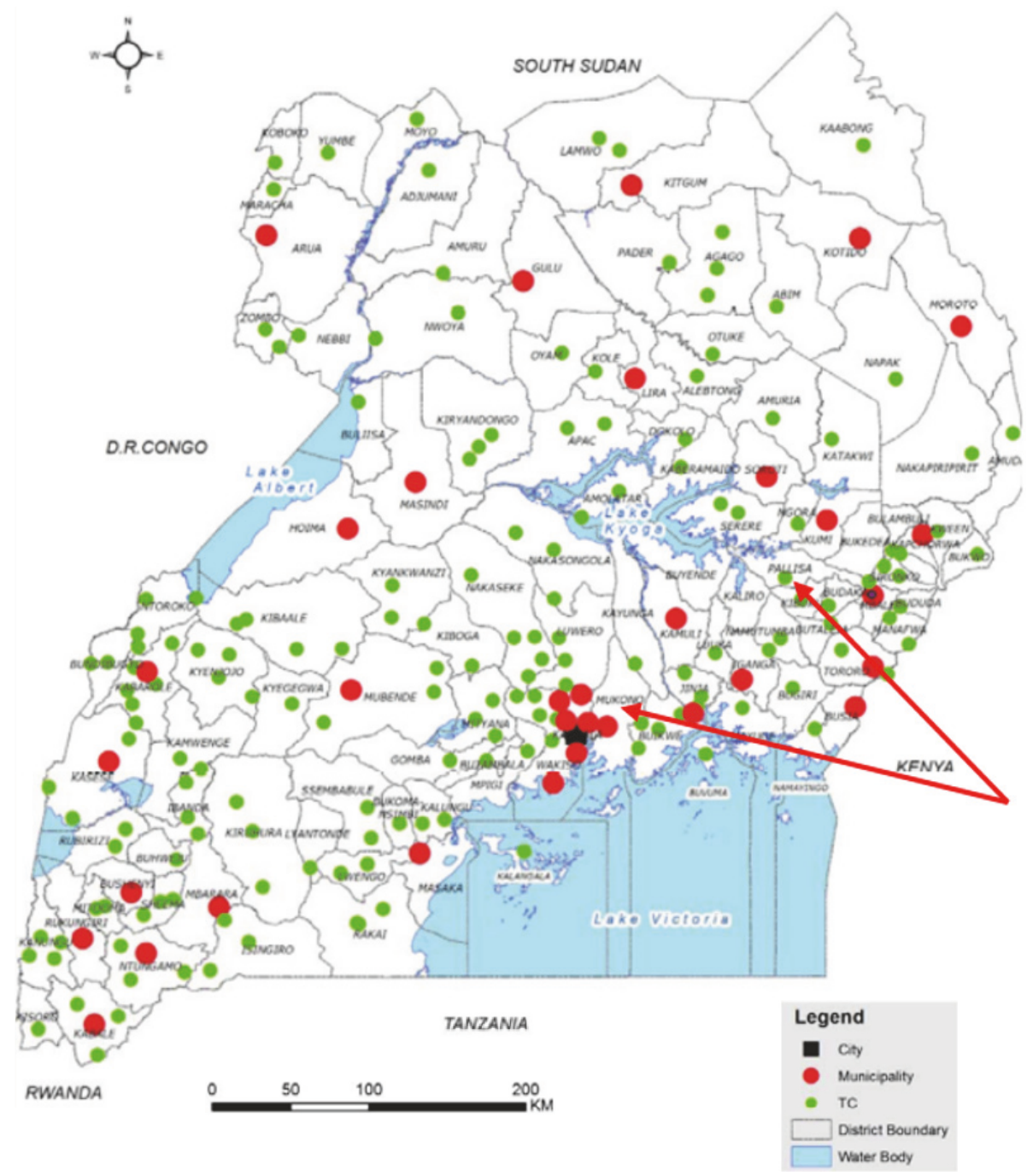

FIgURE 1: Map of Uganda showing Mukono and Pallisa districts.

colour. Some described it as a slippery and sticky substance with no smell. The textural descriptions were a sticky stool that appears like chicken dropping; a leafy like substance; a scrambled egg; or a tiny black mousse. Most mothers described meconium as waste that babies pass out at birth.

The majority felt that the colour and texture of meconium resulted from the food content the baby takes in during gestation or the substances the baby ingests at birth. However, the majority of those that thought meconium was ingested during delivery were not certain of how this occurs.

Some correctly reported that meconium was waste accumulated during gestation that passes out at birth. All key informants were of the view that meconium provides some clues about the health of the child at birth, and notably, meconium with high temperature was attributed to possibilities of a newborn having an infection. Similarly, TBAs mentioned that a strong meconium odour depicted that the newborn had fever associated illnesses.
3.2. Cultural Practices and Beliefs Associated with Meconium. Cultural practices that involve the use of meconium were investigated. Participants reported that meconium is used to prevent stomach discomfort and other ailments, as a means to confirm paternity, to initiate the child into the clan, and to facilitate father and child bonding. These categories are explained further below.

In both Mukono and Pallisa districts, meconium was widely used as a local remedy to alleviate abdominal discomfort that newborns experienced in the first three months of life. Mothers reported that a string made from a piece of cloth is smeared with meconium and then tied around the baby's waist. They believed that this relieves the baby's abdominal discomfort. Sources of such perception within the community were reported mainly by older mothers and TBAs. One TBA interviewed described how the procedure is performed: "All we do (among the Baganda ethnic group), we get a small piece of the baby stool and we wrap it in 
a small piece of paper making a ball shape, we again wrap it in a piece of cloth and then we tie it around the baby's waist so that the baby isn't disturbed by stomach discomfort (enjoka)" (KI_TBA_01).

It was also reported that as an alternative practice, meconium may be smeared on wall surfaces at a birth site. This was also believed to prevent baby abdominal discomfort and to enhance breast feeding.

Another TBA explained, "At the place where the mother has given birth from, I get a small portion of meconium and I smear it on the wall so that the baby does not fail to breast feed because of abdominal discomfort (obwoka)" (KI_TBA_02).

Mothers in Pallisa reported that meconium was a key item in rituals practiced in certain communities preceding the birth of a newborn. Specifically, they reported that meconium is very helpful in paternity verification and thus proof of the mother's fidelity to the husband. For example, they explained that, in some rituals, meconium is mixed in cooked millet and then served to both the baby's mother and the father. If the newborn is a biological child of the woman's partner as reported, it survives. If the mother lied about the paternity, it was believed that the newborn would die during the ritual. This was confirmed by some health workers in the area as well.

After fatherhood confirmation, they proceed with introductory practices that initiate the child into the father's clan. It was further reported that some mothers hide meconium, which they then take to their home villages for other cultural practices, which they did not substantiate.

It was also revealed that some mothers use meconium to facilitate bonding between children and their fathers. A participant noted that to achieve this, mothers get a piece of meconium, which they mix with a delicacy prepared for the child's father. When the father eats, it is believed that his love for the child increases and that such fathers tend to bond better with their children. A VHT member explained, "Women are the ones who use it (meconium) so much because they use it in food or tea and they serve it to their husbands. They say they want to create a close relationship between the father and the child" (KI_VHT-10).

3.3. Meconium Disposal Practices. The mentioned common disposal sites included pit latrines, placenta pits that they use while the baby is still in hospital, the bushes, at open rubbish pits, dig-and-burry holes, and some at dedicated locations in banana plantations in their homes. Cultural beliefs were critical in determining where the disposal took place, with the disposal sites primarily being determined by mothers and their culturally grounded caretakers.

Those who disposed of meconium in pit latrines either were cautious of their household hygiene and sanitation status or had this as their only option. However, from KIs, it was also noted that fear of adversaries influenced choice of disposal into a pit latrine as explained in one of the meetings: "... another thing I know is that they do not want to pour meconium anywhere for reasons that someone may come and do something evil with it and the newborn gets problems. So, whenever they finish washing, they make sure that the water, which contains meconium, is poured into the pit latrine" (KII -Health Worker 8).
However, some participants indicated that it was a taboo in some communities to dispose of meconium into a pit latrine. They highlighted the existence of the following beliefs associated with disposal into a pit latrine: delayed growth of the baby's milk teeth or sometimes total inhibition of their growth; frequent childhood illness; and delayed commencement of breast feeding by the newborn. It was also reported that disposing meconium in pit latrines was equated to placing an omen on the newborn, and it should not be done so as to avoid the negative attributes. One KI explained, "Sometimes they (mothers) say that when a baby's stool mixes with adult faeces the child may develop illnesses. That's why they don't want to mix it with adult faeces to avoid frequent sickness of the newborn baby" (KI_VHT-15).

When asked about the frequent childhood illness experienced, they pointed out abdominal discomfort, "mysterious traditional ailments", back pain, and fever. It was mentioned that it was better to dispose of stool into a pit latrine when the baby has started feeding on solid food.

Although it was recognized as taboo to dispose of meconium into pit latrines by some mothers, others perceived the practice as being protective of the baby especially from witchcraft. They reported that disposal into pit latrines denied access to those adversaries who would use the baby excreta to harm them. "My mother told me that I shouldn't dispose the baby's pooh anyhow; she told me to be throwing it in the pit latrine directly.... Asked why, she told me that it is not good because some people can do something bad with that pooh and your baby ends up falling sick" (IDI- 21).

Some mothers reported that they disposed of meconium at the base of a banana tree within their plantation and that the banana species chosen for this was dependent on the sex of the child.

This way of disposal was perceived to help the child grow with humility and to receive blessings. One KI narrated, “... Yes, they would dig a hole and place it there such that when the child grows up, she/he would be humble and blessed, and not wild" (KII- VHT10).

Mothers whose newborns passed out meconium while at the hospital disposed of the waste into the placenta pit. This was corroborated by KI health workers, who mentioned that they direct mothers to pour the waste in the placenta pit. However, it was stated that those mothers who were superstitious hid and took the meconium home for cultural use as already stated above.

The majority of the young mothers knew of the existence of the several cultural perceptions and practices associated with meconium, although they claimed they did not practice any themselves. Among these, it was reported that modernization and religion were the major reasons for not recognizing any cultural practices related to meconium. To affirm, a TBA reported that “....many mothers don't observe the cultural practices associated with meconium and many don't know the taboos associated with its use and disposal" (KI_TBA_01).

3.4. Influence on Willingness to Provide Meconium for Research Purposes. Mothers were asked about their willingness to provide meconium as a laboratory sample for research to 
inform future studies that could use meconium in investigating environmental exposures.

None of the mothers had ever participated in research that involved meconium collection and to most, the study would be very unusual. However, some indicated willingness to participate.

3.4.1. Perceived Benefits and Fears. The desire to detect possible ailments in their children would be a "pull factor" for mothers to participate and provide meconium for research. The mothers' love for their children's wellbeing was mainly expressed. The mothers indicated that if their newborns are diagnosed with potential for future ailments, healthcare providers would advise them accordingly. One mother reflected, "...maybe she could be having some ailment and they happen to advise me on how to treat the child and it saves me a lot in future" (IDI - 3).

Some mothers reported that they did not have any use for meconium and therefore, giving it away for research purposes would not be a problem. They noted that, instead of disposing of meconium, they would rather provide it for research as long as it would be useful in that perspective.

Although most participants indicated that they would be willing to participate and provide meconium for research purposes, some mothers expressed fears and uncertainties. The fears related to the credibility and intentions of researchers; use of meconium for witchcraft purposes; the feeling that meconium was disgusting to be given to researchers; uncertainties about why meconium was needed for research and what kind of research it would be; and the fears of spouses and/or elders not approving participation.

Some mothers were also afraid that unscrupulous people disguised as researchers could give meconium to sorcerers whose intentions might be to harm children. Mothers and KIs commonly reported fear of sorcery whose communities' sorcery practices were prevalent. A mother narrated, "Now the first challenge is that most mothers in this village believe so much in witchcraft that they would think that there is something wrong you are going to use that meconium for. It is because they hold the same perceptions that they fear you are probably going to do something evil with it. So, should her child fall sick, she will attribute that to the meconium that was collected simply because herself, practices witchcraft. So she may even fail to take the child to hospital for treatment thinking that; 'what I do is what these people also did"' (KI_VHT_10).

3.4.2. Uncertainty and Scepticism about Why Meconium Would Be Needed for Research. The participants noted that meconium was quite a rare specimen that was not requested in the past and therefore, they wondered why of all specimens a researcher would come requesting meconium. Most mothers noted that it was the first time to hear of a study that would request meconium and would therefore feel uncomfortable giving it away.

Some participants felt that meconium was filthy and that they would feel ashamed to participate in such research activities. One of the participants reflected, "The mother can feel ashamed to give someone such things (meconium) because they look bad." (IDI_03).
Mothers were sceptical that their spouses or family elders would not approve of their involvement in such a study. They indicated that they would have to seek authorization from their spouses or family elders, before providing meconium for research purposes.

Participants proposed recommendations to overcome the scepticism, where they indicated that like any other new health programme that faces similar challenges in communities, proper identification, sensitization, and liaison with local community leaders would be paramount in penetrating the community for such a study. It was also suggested that mothers should be informed of the purpose and procedure of the study well ahead of the newborn delivery. They further proposed that researchers would have to use midwives, VHT members, TBAs, and religious, cultural, and political leaders to raise awareness about the harmless nature and purpose of a meconium study. Additionally, they mentioned that researchers should make use of health programmes like antenatal care and immunization to raise awareness about the study. These programmes offer the opportunity to meet mothers in big numbers in a health setting. A participant in Mukono district advised that "You can use midwives to sensitize these people especially during antenatal visits- these can sensitize people about the research and ask the mothers to welcome the research.” (IDI_03).

Another participant added, "They will accept if during antenatal the midwives inform them that when the baby passes out the first stool it will be required for investigation" (IDI_02). They argued that because mothers trust their health workers, it would be easier for samples to be collected by midwives or health workers at health facilities than from the communities. "It is because, they naturally have trust in these health workers even when they have not met them before but they have introduced themselves- like you have done - and you explained to them what they have come to do. Yeah somebody will be willing to give it to you" (KII -Health worker 9). Some mothers suggested that if meconium can be relied on to assess in utero exposures, then the Ministry of Health should make it a policy for mothers to provide meconium as the case is for antenatal care and immunization programmes attendance, so as to identify illnesses early in their babies. One participant views, "It should be a policy that when a baby passes out the first stool you have to give it to the health worker for assessment" (IDI_02).

\section{Discussion}

Our findings show that there exist a variety of perceptions, cultural practices, and beliefs around newborns that would greatly affect the acceptability of mothers to participate in studies assessing environmental exposures through examining meconium. The qualitative methods employed allowed gaining deeper insight into participants' practices, perceptions, and beliefs on meconium, as well as their willingness to participate in future research involving meconium to assess exposure to environmental contaminants. The idea of meconium as a medium that can be used to study in utero exposures to environmental contaminants was new among all the mothers interviewed in this study. This finding was consistent with those reported elsewhere [26]. 
A prevalent belief among mothers that meconium has a number of associated taboos was consistent with findings reported in another study in India [27]. In order to implement the use of meconium in research, there is need to assess knowledge and understanding of cultural perceptions and practices with regard to meconium. In any society, the birth experience is socially constructed, occurring within a cultural context and being shaped by the perceptions and practices of that culture [28]. Therefore, there are many beliefs and practices relating to the childbearing process that the woman and her family must observe to ensure the health and wellbeing of not only herself but also that of her newborn infant [28-30].

The practice of fathers unknowingly ingesting meconium via food or drink is a health hazard that mothers should be discouraged from practicing. Contaminants that are documented to be present in meconium include heavy metals [31$33]$, pesticides $[31,34]$, and phthalates [35, 36]. Therefore, fathers are potentially at risk of exposure to these contaminants. Mothers should be educated about the harmful effects of such a cultural practice that could potentially expose fathers to contaminants.

Addressing the perceptions and beliefs is important and can be tackled through the provision of education and behaviour change interventions. Majority of mothers (73.4\%) had very low level of formal education or none at all, a situation that might present a challenge of how to explain the link between environmental exposures and meconium to such populations. However, education about beliefs may not be affected so much by the levels of education if it is done in the local languages with appropriate contextual factors in consideration. Future educational interventions prior to enrollment of mothers should understand and focus on dissuading misconceived beliefs and myths among the mothers. Although there was meconium related stigma that is multilayered, with cultural issues, fear, and ignorance, some mothers expressed willingness to participate in a study requiring meconium from their newborn infants.

Our findings suggest that involvement of health workers, particularly midwives in the collection of meconium while the newborn is still at the health facility would improve acceptability of mothers to the provision of meconium. Development of trust and confidence between the mothers, health workers, and the researcher would be key to the mothers' participation and consequently the success of a meconium study. Educating the health workers on environmental hazards and the benefit of collecting meconium would facilitate the recruitment process of mothers into studies where meconium is to be utilized [37].

An increasing number of studies have addressed the concern that environmental pollutants may contribute to the early origin of diseases [38-40]. Our findings show that mothers' knowledge that meconium could provide information about the potential of their child being at risk of developing adverse health outcomes would enhance their participation in a meconium study.

\section{Conclusions}

The study revealed that mothers in the two rural districts of Mukono and Pallisa in Uganda have cultural beliefs and practices, which might inhibit their willingness to participate in studies designed to assess environmental exposures using meconium from their babies. It was noted that the mothers' level of willingness to participate in a meconium study could be enhanced through educational and behaviour change interventions from their health workers during the entire pregnancy period. This study also brings forth a cultural practice that may expose the male spouses to environmental contaminants that could be contained in meconium and therefore must be abandoned. Since most mothers indicated willingness to participate in a meconium study if it could inform them of their newborns' health, research in low income countries ought to utilize this research opportunity among mothers.

\section{Data Availability}

Data underlying the findings of the study shall be made available upon request, through the School of Public Health Higher Degrees, Research and Ethics Committee Administrator. The Administrator can be contacted at the email address: wtusiime@musph.ac.ug.

\section{Conflicts of Interest}

The authors have no conflicts of interest to declare.

\section{Authors' Contributions}

John C. Ssempebwa conceived the study and contributed to the design of the study. John C. Ssempebwa, Geofrey Musinguzi, and Simon Peter Sebina Kibira participated in writing and critical review of the manuscript. All authors read and approved the final manuscript.

\section{Acknowledgments}

This study was supported by the research grants from the U.S. National Institute of Health (Grant nos. [1R24TW009489] and [1R24TW009556]).

\section{References}

[1] R. B. Gunier, A. M. Mora, D. Smith et al., "Biomarkers of Manganese Exposure in Pregnant Women and Children Living in an Agricultural Community in California," Environmental Science \& Technology, vol. 48, no. 24, pp. 14695-14702, 2014.

[2] M. Ratelle, J. Coté, and M. Bouchard, "Time profiles and toxicokinetic parameters of key biomarkers of exposure to cypermethrin in orally exposed volunteers compared with previously available kinetic data following permethrin exposure," Journal of Applied Toxicology, vol. 35, no. 12, pp. 1586-1593, 2015.

[3] S. T. Singleton, P. J. Lein, O. A. Dadson et al., "Longitudinal assessment of occupational exposures to the organophosphorous insecticides chlorpyrifos and profenofos in Egyptian cotton field workers," International Journal of Hygiene and Environmental Health, vol. 218, no. 2, pp. 203-211, 2015.

[4] G. Talaska, J. Thoroman, B. Schuman, and H. U. Käfferlein, "Biomarkers of polycyclic aromatic hydrocarbon exposure in 
European coke oven workers," Toxicology Letters, vol. 231, no. 2, pp. 213-216, 2014.

[5] K. F. Arcaro and D. L. Anderton, "Potential of using breast milk as a tool to study breast cancer and breast cancer risk," Future Oncology, vol. 4, no. 5, pp. 595-597, 2008.

[6] A. J. Wheeler, N. A. Dobbin, M.-E. Héroux et al., "Urinary and breast milk biomarkers to assess exposure to naphthalene in pregnant women: An investigation of personal and indoor air sources," Environmental Health: A Global Access Science Source, vol. 13, no. 1, article no. 30, 2014.

[7] K. Delano and G. Koren, "Emerging Biomarkers of Intrauterine Neonatal and Pediatric Exposures to Xenobiotics," Pediatric Clinics of North America, vol. 59, no. 5, pp. 1059-1070, 2012.

[8] D. Chan, D. Caprara, P. Blanchette, J. Klein, and G. Koren, "Recent developments in meconium and hair testing methods for the confirmation of gestational exposures to alcohol and tobacco smoke," Clinical Biochemistry, vol. 37, no. 6, pp. 429438, 2004.

[9] Z. Hong, M. Günter, and F. F. E. Randow, "Meconium: A matrix reflecting potential fetal exposure to organochlorine pesticides and its metabolites," Ecotoxicology and Environmental Safety, vol. 51, no. 1, pp. 60-64, 2002.

[10] E. M. Ostrea Jr., D. M. Bielawski, N. C. Posecion Jr. et al., "A comparison of infant hair, cord blood and meconium analysis to detect fetal exposure to environmental pesticides," Environmental Research, vol. 106, no. 2, pp. 277-283, 2008.

[11] R. M. Whyatt and D. B. Barr, "Measurement of organophosphate metabolites in postpartum meconium as a potential biomarker of prenatal exposure: A validation study," Environmental Health Perspectives, vol. 109, no. 4, pp. 417-420, 2001.

[12] C. F. Bearer, "Meconium as a biological marker of prenatal exposure," Academic Pediatrics, vol. 3, no. 1, pp. 40-43, 2003.

[13] E. M. Ostrea Jr., D. M. Bielawski, N. C. Posecion Jr. et al., "Combined analysis of prenatal (maternal hair and blood) and neonatal (infant hair, cord blood and meconium) matrices to detect fetal exposure to environmental pesticides," Environmental Research, vol. 109, no. 1, pp. 116-122, 2009.

[14] G. Turker, G. Özsoy, S. Özdemir, B. Barutçu, and A. S. Gökalp, "Effect of heavy metals in the meconium on preterm mortality: Preliminary study," Pediatrics International, vol. 55, no. 1, pp. 30-34, 2013.

[15] H.-C. Hsi, C.-B. Jiang, T.-H. Yang, and L.-C. Chien, "The neurological effects of prenatal and postnatal mercury/methylmercury exposure on three-year-old children in taiwan," Chemosphere, vol. 100, pp. 71-76, 2014.

[16] S. Narkowicz, Z. Polkowska, B. Kielbratowska, and J. Namiesnik, "Meconium Samples use to assess infant exposure to the components of ETS during pregnancy," International Journal of Occupational Medicine and Environmental Health, vol. 28, no. 6, pp. 955-970, 2015.

[17] M. McNeilly, M. Musick, and J. R. Efland, "Minority populations and psychophysiological research: challenges in trust building and recruitment," Journal of Mental Health and Aging, vol. 6, pp. 91-102, 2000.

[18] L. Marcellus, "Is meconium screening appropriate for universal use? Science and ethics say no," Advances in Neonatal Care, vol. 7, no. 4, pp. 207-214, 2007.

[19] S. Neelotpol, A. W. M. Hay, A. J. Jolly, and M. W. Woolridge, "Challenges in collecting clinical samples for research from pregnant women of South Asian origin: evidence from a UK study," BMJ Open, vol. 6, no. 8, p. e010554, 2016.
[20] L. C. Callister, M. N. Eads, and J. P. Yeung Diehl, "Perceptions of Giving Birth and Adherence to Cultural Practices in Chinese Women," MCN, The American Journal of Maternal/Child Nursing, vol. 36, no. 6, pp. 387-394, 2011.

[21] E. Naser, S. Mackey, D. Arthur, P. Klainin-Yobas, H. Chen, and D. K. Creedy, "An exploratory study of traditional birthing practices of Chinese, Malay and Indian women in Singapore," Midwifery, vol. 28, no. 6, pp. e865-e871, 2012.

[22] L. M. Vallely, P. Homiehombo, A. Kelly-Hanku, A. Vallely, C. S. E. Homer, and A. Whittaker, "Childbirth in a rural highlands community in Papua New Guinea: A descriptive study, Midwifery, vol. 31, no. 3, pp. 380-387, 2015.

[23] I. Zelner, S. Shor, H. Lynn et al., "Neonatal screening for prenatal alcohol exposure: Assessment of voluntary maternal participation in an open meconium screening program," Alcohol, vol. 46, no. 3, pp. 269-276, 2012.

[24] K. E. Wood, L. L. Sinclair, C. D. Rysgaard, F. G. Strathmann, G. A. McMillin, and M. D. Krasowski, "Retrospective analysis of the diagnostic yield of newborn drug testing," BMC Pregnancy and Childbirth, vol. 14, no. 1, article no. 250, 2014.

[25] N. Mohammadi, T. Jones, and D. Evans, "Participant recruitment from minority religious groups: The case of the Islamic population in South Australia," International Nursing Review, vol. 55, no. 4, pp. 393-398, 2008.

[26] G. Koren, J. Hutson, and J. Gareri, "Novel methods for the detection of drug and alcohol exposure during pregnancy: Implications for maternal and child health," Clinical Pharmacology \& Therapeutics, vol. 83, no. 4, pp. 631-634, 2008.

[27] U. K. Choudhry, "Traditional practices of women from India: pregnancy, childbirth, and newborn care.", Journal of Obstetric, Gynecologic, \& Neonatal Nursing, vol. 26, no. 5, pp. 533-539, 1997.

[28] S. Steinberg, "Childbearing research: A transcultural review," Social Science \& Medicine, vol. 43, no. 12, pp. 1765-1784, 1996.

[29] B. Jordan, "Authoritative knowledge and its construction," in Childbirth and Authoritative Knowledge: Cross-Cultural Perspectives, R. E. Davis-Floyd and C. F. Sargent, Eds., University of California Press, Berkeley, California, CA, USA, 1997.

[30] P. Liamputtong, S. Yimyam, S. Parisunyakul, C. Baosoung, and N. Sansiriphun, "Traditional beliefs about pregnancy and child birth among women from Chiang Mai, Northern Thailand," Midwifery, vol. 21, no. 2, pp. 139-153, 2005.

[31] E. M. Ostrea, V. Morales, E. Ngoumgna et al., "Prevalence of fetal exposure to environmental toxins as determined by meconium analysis," NeuroToxicology, vol. 23, no. 3, pp. 329-339, 2002.

[32] C.-B. Jiang, H.-C. Hsi, C.-H. Fan, and L.-C. Chien, "Fetal exposure to environmental neurotoxins in Taiwan," PLoS ONE, vol. 9, no. 10, Article ID 0109984, 2014.

[33] S. Peng, L. Liu, X. Zhang et al., "A nested case-control study indicating heavy metal residues in meconium associate with maternal gestational diabetes mellitus risk," Environmental Health: A Global Access Science Source, vol. 14, no. 1, article no. 19, 2015.

[34] T. Berton, F. Mayhoub, K. Chardon et al., "Development of an analytical strategy based on LC-MS/MS for the measurement of different classes of pesticides and theirs metabolites in meconium: Application and characterisation of foetal exposure in France," Environmental Research, vol. 132, pp. 311-320, 2014.

[35] K. Kato, M. J. Silva, L. L. Needham, and A. M. Calafat, "Quantifying phthalate metabolites in human meconium and semen 
using automated off-line solid-phase extraction coupled with on-line SPE and isotope-dilution high-performance liquid chromatography-tandem mass spectrometry," Analytical Chemistry, vol. 78, no. 18, pp. 6651-6655, 2006.

[36] C. Xie, R. Jin, Y. Zhao et al., "Paraoxonase 2 gene polymorphisms and prenatal phthalates' exposure in Chinese newborns," Environmental Research, vol. 140, pp. 354-359, 2015.

[37] M. Ondeck and J. Focareta, "Environmental Hazards Education for Childbirth Educators," Journal of Perinatal Education, vol. 18, no. 4, pp. 31-40, 2009.

[38] T. E. Arbuckle, "Maternal-infant biomonitoring of environmental chemicals: The epidemiologic challenges," Birth Defects Research Part A: Clinical and Molecular Teratology, vol. 88, no. 10, pp. 931-937, 2010.

[39] G. E. R. Schoeters, E. Den Hond, G. Koppen et al., "Biomonitoring and biomarkers to unravel the risks from prenatal environmental exposures for later health outcomes," American Journal of Clinical Nutrition, vol. 94, no. 6, 2011.

[40] T. Fernández-Cruz, E. Martínez-Carballo, and J. Simal-Gándara, "Perspective on pre- and post-natal agro-food exposure to persistent organic pollutants and their effects on quality of life," Environment International, vol. 100, pp. 79-101, 2017. 


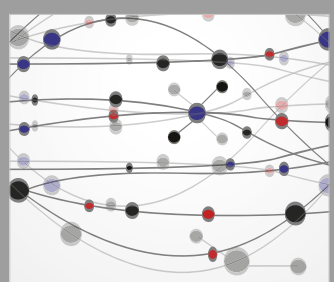

The Scientific World Journal
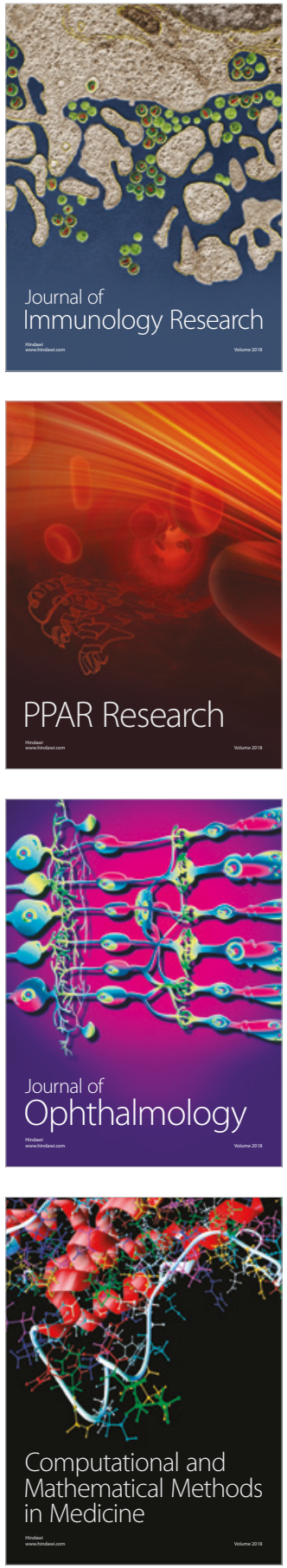

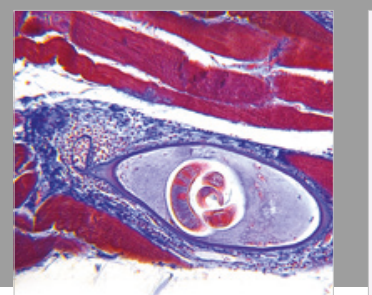

Gastroenterology Research and Practice

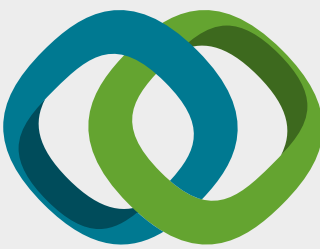

\section{Hindawi}

Submit your manuscripts at

www.hindawi.com
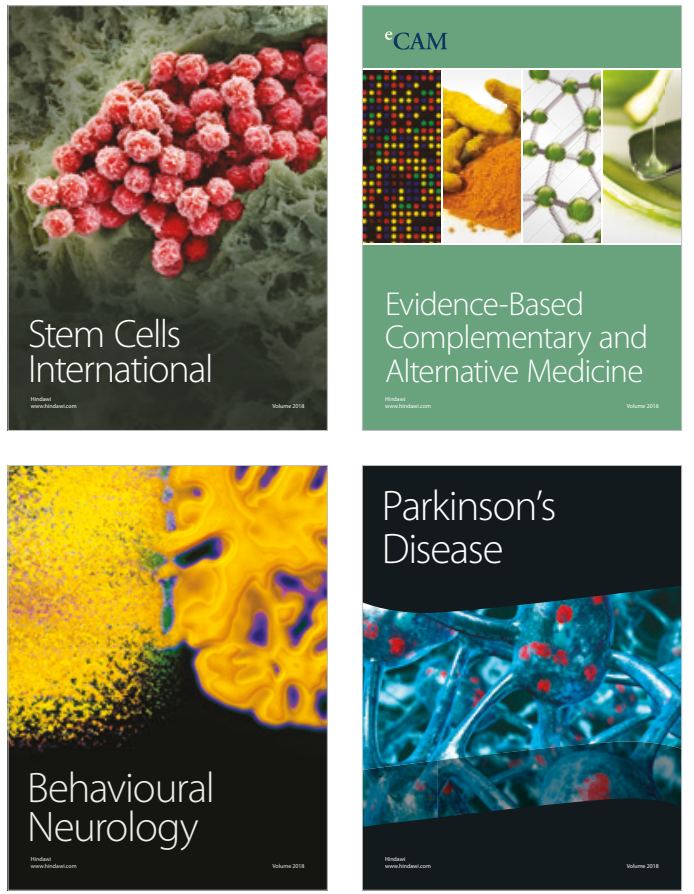

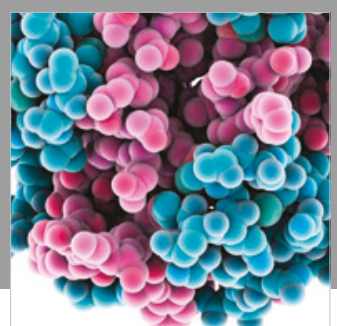

ournal of

Diabetes Research

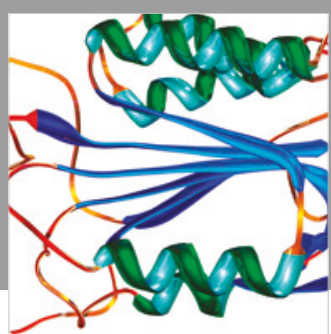

Disease Markers
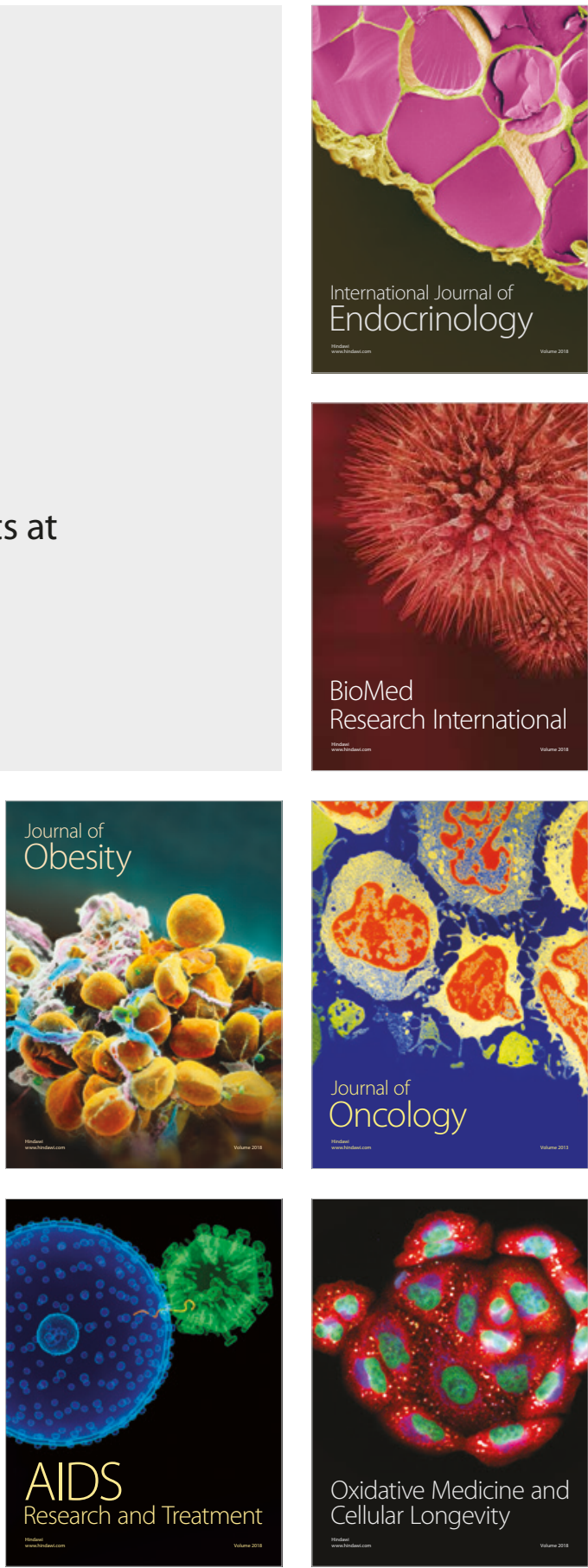